\title{
Laparoscopic versus open pancreaticoduodenectomy with major vein resection for pancreatic head cancer: A single surgeon's experience
}

Min Kyu SUNG, Ki Byung SONG*, Yejong PARK, Jaewoo KWON, Woohyung LEE, Jae Hoon LEE, Dae Wook HWANG, Song Cheol KIM

Division of Hepatobiliary and Pancreatic Surgery, Department of Surgery, Asan Medical Center, Seoul, Korea

Introduction: Laparoscopic pancreaticoduodenectomy with major vein resection is a challenging procedure. We aimed to evaluate the feasibility and safety of laparoscopic vein resection in pancreatic head cancer with portal/superior mesenteric vein invasion and compared differences in the survival of laparoscopic surgery with that of open surgery. Additionally, we assessed prognostic factors for disease-free survival in patients with pancreatic head cancer with major vein invasion.

Methods: We retrospectively reviewed the electronic medical records of all patients whose surgery was performed by a single surgeon from January 2016 to December 2017. We plotted the Kaplan-Meier curve to compare disease-free survival between patients who underwent laparoscopic surgery and open surgery. We used Cox-proportional hazard models to analyze prognostic factors for survival. Results: Among a total of 53 patients, 41 patients had open surgery, and 12 patients had laparoscopic surgery. There was no significant difference in complication rates including portal vein stenosis and portal vein thrombus, recurrence of tumors, and pathological outcomes after surgery. There was no significant difference in disease-free survival $(p=0.541)$ between the two groups. Additionally, the surgery method was not an independent prognostic factor for pancreatic head cancer with major vein invasion after adjusting for potential confounders.

Conclusions: The disease-free survival and complication outcomes were not inferior in laparoscopic surgery compared with open surgery. Surgery method was not an independent prognostic factor for pancreatic head cancer with major vein invasion in our analysis. 